\title{
Vitamin D supplementation and mortality risk in chronic kidney disease: a meta-analysis of 20 observational studies
}

\author{
Zhenfeng Zheng ${ }^{1}$, Huilan Shi ${ }^{2}$, Junya Jia ${ }^{1}$, Dong Li ${ }^{1}$ and Shan Lin ${ }^{1 *}$
}

\begin{abstract}
Background: Vitamin D insufficiency correlates with mortality risk among patients with chronic kidney disease (CKD). The survival benefits of active vitamin $D$ treatment have been assessed in patients with CKD not requiring dialysis and in patients with end stage renal disease (ESRD) requiring dialysis.

Methods: MEDLINE, Embase, the Cochrance Library, and article reference lists were searched for relevant observational trials. The quality of the studies was evaluated using the Newcastle-Ottawa Scale (NOS) checklist. Pooled effects were calculated as hazard ratios (HR) using random-effects models.

Results: Twenty studies (11 prospective cohorts, 6 historical cohorts and 3 retrospective cohorts) were included in the meta-analysis., Participants receiving vitamin D had lower mortality compared to those with no treatment (adjusted case mixed baseline model: HR, 0.74; 95\% confidence interval [95\% Cl], 0.67-0.82; $P<0.001$; timedependent Cox model: $\mathrm{HR}, 0.71 ; 95 \% \mathrm{Cl}, 0.57-0.89 ; P<0.001)$. Participants that received calcitriol $(\mathrm{HR}, 0.63 ; 95 \% \mathrm{Cl}$, 0.50-0.79; $P<0.001)$ and paricalcitol (HR, $0.4395 \% \mathrm{Cl}, 0.29-0.63 ; P<0.001)$ had a lower cardiovascular mortality. Patients receiving paricalcitol had a survival advantage over those that received calcitriol $(\mathrm{HR}, 0.95 ; 95 \% \mathrm{Cl}, 0.91-0.99 ; P<0.001)$.

Conclusions: Vitamin D treatment was associated with decreased risk of all-cause and cardiovascular mortality in patients with CKD not requiring dialysis and patients with end stage renal disease (ESRD) requiring dialysis. There was a slight difference in survival depending on the type of vitamin D analogue. Well-designed randomized controlled trials are necessary to assess the survival benefits of vitamin D.
\end{abstract}

Keywords: Vitamin D, Mortality, Calcitriol, Paricalcitol, Chronic kidney disease

\section{Background}

Mineral and bone disorders (MBD) are early and common complications of CKD, and progress as glomerular filtration rate (GFR) declines. Multiple factors contribute to the development and maintenance of CKD-MBD, but principally involve phosphate retention and vitamin D metabolism abnormalities. Kidney Disease: Improving Global Outcomes defines chronic kidney disease-mineral and bone disorder (CKD-MBD) as a systemic syndrome characterized by abnormalities in serum calcium, phosphorus and parathyroid hormone $(\mathrm{PTH})$ concentration, vitamin D metabolism, and bone turnover [1]. This syndrome is common among CKD

\footnotetext{
*Correspondence: linshan@medmail.com.cn

${ }^{1}$ Nephrology Department, General Hospital of Tianjin Medical University, No. 154 Anshan Road, Heping District, Tianjin 300052, China
}

patients and has been associated with an increased risk of cardiovascular calcification [2,3] and mortality [4]. The Third National Health and Nutrition Examination Survey (NHANES III) reported 15068 adults patients with vitamin $\mathrm{D}$ deficiency and demonstrated a higher prevalence of cardiovascular disease and mortality in untreated patients [5]. An association between vitamin D deficiency and other traditional cardiovascular risk factors, such as hypertension, insulin resistance, diabetes, and dyslipidemia, has also been reported [6,7]. The recognition of biochemical components of CKD-MBD associated with increased mortality in dialysis patients [8] and in patients with CKD not treated with dialysis [9] has provided an impetus to explore the effect of these factors on survival and associated treatment modalities. Numerous reports have characterized the nonskeletal benefits of vitamin D [10].
Full list of author information is available at the end of the article

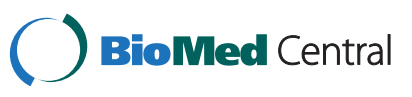


Wang et al. and Pittas et al. reported the benefits of vitamin D supplementation on cardiovascular disease (CVD) in the general population [11,12]. Nutritional vitamin $\mathrm{D}$ supplementation has also been reported to be beneficial to CKD patients [13]. Most reviews, however, had few participants, short follow-up, and lacked survival analyses. We conducted a systematic review of the literature to assess whether vitamin D supplements reduced mortality in patients with ESRD on dialysis and patients with CKD not requiring dialysis.

\section{Methods}

Data sources and Search strategy

MEDLINE (1966 to March 2013), EMBASE (1980 to March 2013) and the Cochrance Controlled Trials Register (CCTR-Specialized Renal Registry) were searched. Relevant studies were identified [14,15]. References from identified studies were reviewed to find additional relevant studies. This systematic review was planned, conducted, and reported following the Meta-analysis of Observational Studies in Epidemiology (MOOSE) guidelines [16].

\section{Eligibility criteria}

Studies were included in the meta-analysis if they met the following criteria: (1) cohort study design and follow-up duration was at least 1 year; (2) patients had chronic kidney disease or renal replacement treatment; (3) patients were treated with active vitamin D sterols (alfacalcidol, doxercalciferol, calcitriol, maxacalcitol, falecalcitriol and paricalcitol), but not native vitamin D (ergocalciferol and cholecalciferol); (4) the outcome of interest was all-cause mortality or cardiovascular mortality; (5) there was quantitative data (i.e., events rates, risk ratio [RR] or hazard ratio $[\mathrm{HR}])$. If data were duplicated in more than 1 study, we included the study with the largest number of patients.

\section{Data extraction}

All data were independently abstracted by 2 investigators (Z.F.Z. and H.L.S) using a standardized data collection form. Discrepancies were resolved through discussion with other investigators (D.L. and J.Y.J.) and through reference to the original articles.

\section{Quality assessment}

Two authors (Z.F.Z. and H.L.S.) independently evaluated the quality of each study using the 9-star Newcastle-Ottawa Scale (NOS) [17]. The Strengthening the Reporting of Observational Studies in Epidemiology (STROBE) checklist for cohort studies was used to limit heterogeneity resulting from differences in study design [18]. Disagreements were resolved by consensus.

\section{Statistical analysis}

Studies that provided relative risk (RR) or hazard ratios (HR) were used directly in the pooled meta-analysis calculations. Overall crude (unadjusted) $\mathrm{HR}$ and adjusted $\mathrm{HR}$ were calculated. Adjusted variables included demographic and clinical values, biochemical indices and erythropoietin and phosphate binder use. The overall pooled-effect estimates were calculated using DerSimonian \& Laird randomeffect models. The $\mathrm{Q}$ test was used to assess the presence of heterogeneity and the $\mathrm{I}^{2}$ index was used to quantify the extent of heterogeneity $[19,20] . \mathrm{I}^{2}$ values of $25 \%$ or less indicated low heterogeneity, values near $50 \%$ indicated moderate heterogeneity, and values $75 \%$ or greater indicated high heterogeneity [21]. Publication bias was assessed using funnel plots for each outcome and $\ln$

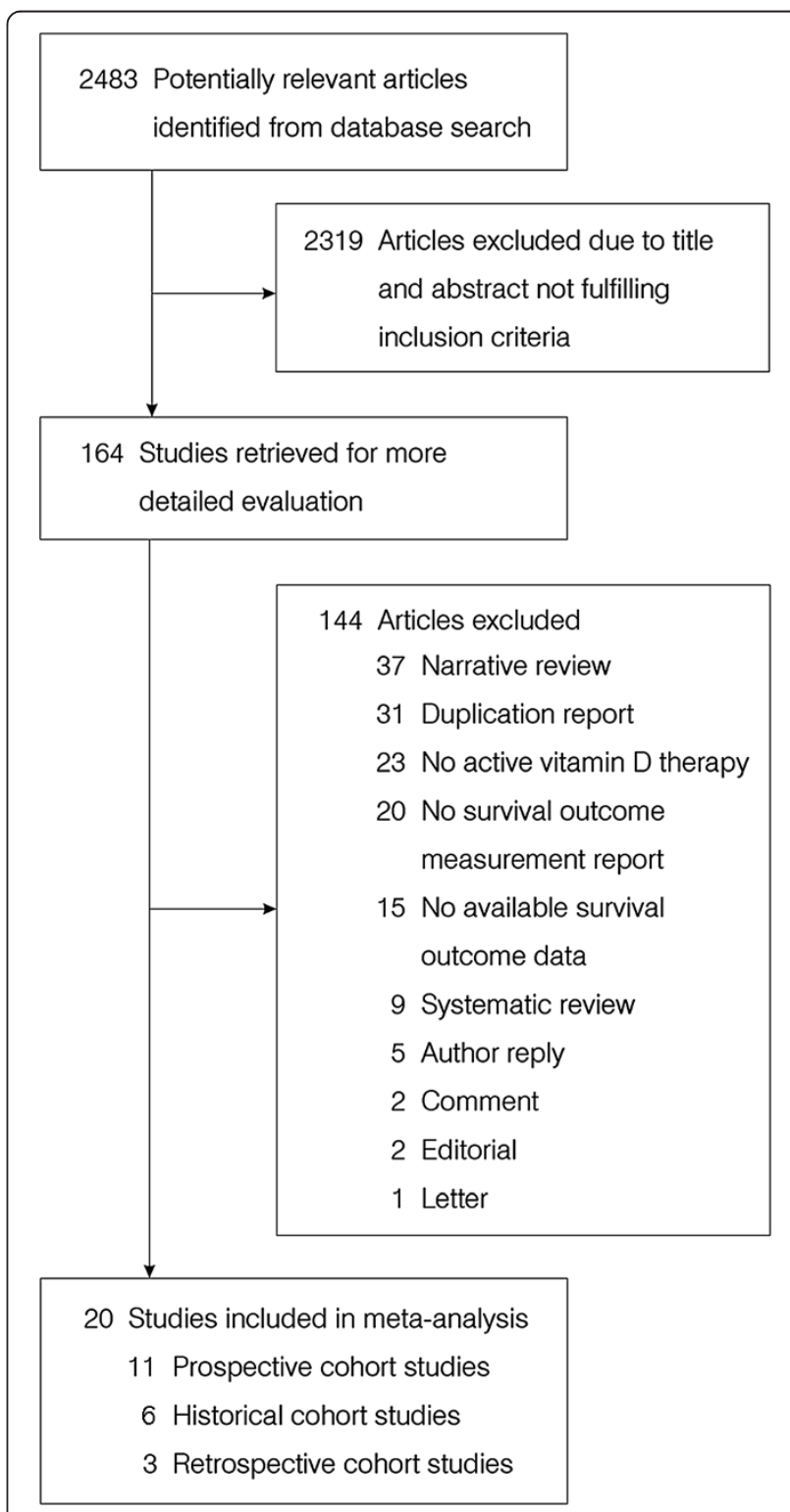

Figure 1 Selection process for studies included in the meta-analysis. 
Table 1 Observational studies examining active vitamin D administration in patients with CKD or on dialysis

\begin{tabular}{|c|c|c|c|c|c|c|c|c|c|c|c|c|c|}
\hline Author & Year & Country & \# Participants & $\begin{array}{l}\text { Study } \\
\text { period }\end{array}$ & $\begin{array}{l}\text { Patient } \\
\text { category }\end{array}$ & Treatment & Comparator & $\begin{array}{l}\text { Vitamin D } \\
\text { dosage }\end{array}$ & $\begin{array}{l}\text { Study } \\
\text { design }\end{array}$ & $\begin{array}{l}\text { Statistical } \\
\text { methods }\end{array}$ & $\begin{array}{l}\text { Follow-up } \\
\text { duration } \\
\text { months }\end{array}$ & $\begin{array}{l}\text { ITT } \\
\text { analysis }\end{array}$ & $\begin{array}{l}\text { NOS } \\
\text { scale }\end{array}$ \\
\hline Teng et al. & 2003 & United States & 67399 & 1999 to 2001 & $\begin{array}{l}\text { Prevalent } \\
\text { HD patients }\end{array}$ & Paricalcitol & Calcitriol & NA & $\begin{array}{l}\text { Historical cohort } \\
\text { multicenter } \\
\text { study }\end{array}$ & $\begin{array}{l}\text { Baseline Cox } \\
\text { model; as- } \\
\text { treated analysis }\end{array}$ & 36 & no & 6 \\
\hline Shoji et al. & 2004 & Japan & 242 & 1992 to 1998 & $\begin{array}{l}\text { Prevalent } \\
\text { HD patients }\end{array}$ & Alfacalcidol & $\begin{array}{l}\text { No } \\
\text { treatment }\end{array}$ & NA & $\begin{array}{l}\text { Prospective } \\
\text { cohort single } \\
\text { center study }\end{array}$ & $\begin{array}{l}\text { Baseline Cox } \\
\text { model }\end{array}$ & 76 & no & 9 \\
\hline Teng et al. & 2005 & United States & 51037 & 1996 to 1999 & $\begin{array}{l}\text { Prevalent } \\
\text { HD patients }\end{array}$ & $\begin{array}{l}\text { Calcitriol or } \\
\text { paricalcitol }\end{array}$ & $\begin{array}{l}\text { No } \\
\text { treatment }\end{array}$ & NA & $\begin{array}{l}\text { Historical cohort } \\
\text { multicenter } \\
\text { study }\end{array}$ & $\begin{array}{l}\text { Time-dependent } \\
\text { Cox model; } \\
\text { marginal structural } \\
\text { model }\end{array}$ & 24 & yes & 6 \\
\hline Melamed et al. & 2006 & United States & 1007 & 1995 to 1998 & $\begin{array}{l}\text { Incident } \\
\text { HD and } \\
\text { PD patients }\end{array}$ & Calcitriol & $\begin{array}{l}\text { No } \\
\text { treatment }\end{array}$ & NA & $\begin{array}{l}\text { Prospective } \\
\text { cohort } \\
\text { multicenter } \\
\text { study }\end{array}$ & $\begin{array}{l}\text { Baseline and } \\
\text { time-dependent } \\
\text { Cox models }\end{array}$ & 48 & no & 5 \\
\hline Kalantar-Zadeh et al. & 2006 & United States & 58058 & 2001 to 2003 & $\begin{array}{l}\text { Prevalent } \\
\text { HD patients }\end{array}$ & Paricalcitol & $\begin{array}{l}\text { No } \\
\text { treatment }\end{array}$ & NA & $\begin{array}{l}\text { Historical cohort } \\
\text { multicenter } \\
\text { study }\end{array}$ & $\begin{array}{l}\text { Baseline and } \\
\text { time-dependent } \\
\text { Cox models }\end{array}$ & 24 & no & 7 \\
\hline Tentori et al. & 2006 & United States & 14967 & 1999 to 2004 & $\begin{array}{l}\text { Prevalent } \\
\text { HD patients }\end{array}$ & $\begin{array}{l}\text { Calcitriol; } \\
\text { paricalcitol; } \\
\text { doxercalciferol }\end{array}$ & $\begin{array}{l}\text { No } \\
\text { treatment; } \\
\text { each other }\end{array}$ & NA & $\begin{array}{l}\text { Historical cohort } \\
\text { multicenter } \\
\text { study }\end{array}$ & $\begin{array}{l}\text { Baseline and } \\
\text { time-dependent } \\
\text { Cox models; as } \\
\text { treated analysis }\end{array}$ & 60 & yes & 7 \\
\hline Kovesdy et al. & 2008 & United States & 520 & 1990 to 2007 & $\begin{array}{l}\text { CKD stage } 2 \\
\text { to } 4 \text { patients }\end{array}$ & Calcitriol & $\begin{array}{l}\text { No } \\
\text { treatment }\end{array}$ & $\begin{array}{l}\text { 1.75-3.5 } \\
\text { ug/week }\end{array}$ & $\begin{array}{l}\text { Prospective } \\
\text { cohort single } \\
\text { center study }\end{array}$ & $\begin{array}{l}\text { Baseline Cox } \\
\text { model }\end{array}$ & 48 & no & 6 \\
\hline Naves-Diaz et al. & 2008 & $\begin{array}{l}\text { Argentina; Brazil; } \\
\text { Colombia; Chile; } \\
\text { Mexico; Venezuela }\end{array}$ & 16004 & 2000 to 2004 & $\begin{array}{l}\text { Prevalent } \\
\text { HD patients }\end{array}$ & $\begin{array}{l}\text { Calcitriol or } \\
\text { alfacalcidol }\end{array}$ & $\begin{array}{l}\text { No } \\
\text { treatment }\end{array}$ & NA & $\begin{array}{l}\text { Historical cohort } \\
\text { multicenter } \\
\text { study }\end{array}$ & $\begin{array}{l}\text { Time-dependent } \\
\text { Cox model }\end{array}$ & 54 & yes & 6 \\
\hline Shinaberger et al. & 2008 & United States & 34307 & 2001 to 2004 & $\begin{array}{l}\text { Prevalent } \\
\text { HD patients }\end{array}$ & Paricalcitol & $\begin{array}{l}\text { No } \\
\text { treatment }\end{array}$ & $\begin{array}{l}\text { 1.7-30.8 } \\
\text { ug/week }\end{array}$ & $\begin{array}{l}\text { Historical cohort } \\
\text { multicenter } \\
\text { study }\end{array}$ & $\begin{array}{l}\text { Baseline Cox } \\
\text { model }\end{array}$ & 30 & no & 7 \\
\hline Shoben et al. & 2008 & United States & 1418 & 1999 to 2007 & $\begin{array}{l}\text { CKD stage } \\
3 \text { to } 4 \\
\text { patients }\end{array}$ & Calcitriol & $\begin{array}{l}\text { No } \\
\text { treatment }\end{array}$ & & $\begin{array}{l}\text { Historical cohort } \\
\text { multicenter } \\
\text { study }\end{array}$ & $\begin{array}{l}\text { Baseline Cox } \\
\text { model; as-treated } \\
\text { analysis }\end{array}$ & 48 & yes & 8 \\
\hline Wolf et al. & 2008 & United States & 9303 & 2004 to 2005 & $\begin{array}{l}\text { Incident } \\
\text { HD patients }\end{array}$ & $\begin{array}{l}\text { Calcitriol; } \\
\text { paricalcitol; } \\
\text { doxercalciferol }\end{array}$ & $\begin{array}{l}\text { No } \\
\text { treatment; } \\
\text { stratified } \\
\text { by race }\end{array}$ & NA & $\begin{array}{l}\text { Prospective } \\
\text { cohort } \\
\text { multicenter } \\
\text { study }\end{array}$ & $\begin{array}{l}\text { Baseline Cox } \\
\text { model }\end{array}$ & 12 & no & 5 \\
\hline
\end{tabular}


Table 1 Observational studies examining active vitamin D administration in patients with CKD or on dialysis (Continued)

\begin{tabular}{|c|c|c|c|c|c|c|c|c|c|c|c|c|c|}
\hline Tentori et al. & 2009 & $\begin{array}{l}\text { France; Germany; } \\
\text { Italy; Japan; Spain; } \\
\text { United Kingdom; } \\
\text { United States; } \\
\text { Australia; Belgium; } \\
\text { Canada; New } \\
\text { Zealand; Sweden }\end{array}$ & 38066 & 1996 to 2009 & $\begin{array}{l}\text { Incident } \\
\text { HD patients }\end{array}$ & $\begin{array}{l}\text { Calcitriol; } \\
\text { paricalcitol; } \\
\text { doxercalciferol }\end{array}$ & $\begin{array}{l}\text { No } \\
\text { treatment; } \\
\text { each other }\end{array}$ & NA & $\begin{array}{l}\text { Prospective } \\
\text { cohort } \\
\text { multicenter } \\
\text { study }\end{array}$ & $\begin{array}{l}\text { Baseline and } \\
\text { time-dependent } \\
\text { Cox models; } \\
\text { Marginal } \\
\text { structural model }\end{array}$ & 30 & no & 5 \\
\hline Peter et al. & 2009 & United States & 193830 & 1999 to 2004 & $\begin{array}{l}\text { Prevalent and } \\
\text { incident HD }\end{array}$ & $\begin{array}{l}\text { Calcitriol; } \\
\text { paricalcitol; } \\
\text { doxercalciferol }\end{array}$ & $\begin{array}{l}\text { No } \\
\text { treatment }\end{array}$ & $\begin{array}{l}0.25-3.5 \\
\text { ug/week* }\end{array}$ & $\begin{array}{l}\text { Historical cohort } \\
\text { multicenter } \\
\text { study }\end{array}$ & $\begin{array}{l}\text { Time-dependent } \\
\text { Cox model }\end{array}$ & 63 & no & 6 \\
\hline Chang et al. & 2009 & Taiwan & 702 & 1993 to 2004 & Incident HD & Calcitriol & $\begin{array}{l}\text { No } \\
\text { treatment }\end{array}$ & $\begin{array}{l}\text { 0.75-6.0 } \\
\text { ug/week }\end{array}$ & $\begin{array}{l}\text { Retrospective } \\
\text { cohort single } \\
\text { center study }\end{array}$ & $\begin{array}{l}\text { Baseline Cox } \\
\text { model }\end{array}$ & 140 & no & 6 \\
\hline Konta et al. & 2010 & Japan & 466 & 2003 to 2008 & Incident HD & $\begin{array}{l}\text { Calcitriol; } \\
\text { falecalcitriol; } \\
\text { maxacalcitol }\end{array}$ & $\begin{array}{l}\text { No } \\
\text { treatment }\end{array}$ & $\begin{array}{l}\text { 1.1-5.1 } \\
\text { ug/week; } \\
\text { 1.4-2.6 } \\
\text { ug/week; } \\
\text { 2.6-5.4 } \\
\text { ug/week }\end{array}$ & $\begin{array}{l}\text { Prospective } \\
\text { cohort } \\
\text { multicenter } \\
\text { study }\end{array}$ & $\begin{array}{l}\text { Baseline Cox } \\
\text { model }\end{array}$ & 60 & no & 7 \\
\hline Sugiura et al. & 2010 & Japan & 665 & 1992 to 2008 & Incident HD & Alfacalcidol & $\begin{array}{l}\text { No } \\
\text { treatment }\end{array}$ & $\begin{array}{l}1.75-3.5 \\
\text { ug/week }\end{array}$ & $\begin{array}{l}\text { Retrospective } \\
\text { cohort single } \\
\text { center study }\end{array}$ & $\begin{array}{l}\text { Baseline Cox } \\
\text { model }\end{array}$ & 132 & no & 6 \\
\hline Jean et al. & 2011 & France & 648 & 2005 to 2009 & $\begin{array}{l}\text { Prevalent } \\
\text { HD patients }\end{array}$ & Alfacalcidol & $\begin{array}{l}\text { No } \\
\text { treatment }\end{array}$ & $\begin{array}{l}1.75-9 \\
\text { ug/week }\end{array}$ & $\begin{array}{l}\text { Prospective } \\
\text { cohort } \\
\text { multicenter } \\
\text { study }\end{array}$ & $\begin{array}{l}\text { Baseline Cox } \\
\text { model }\end{array}$ & 42 & no & 5 \\
\hline Brancaccio et al. & 2011 & Italy & 2378 & 2006 to 2007 & $\begin{array}{l}\text { Incident HD } \\
\text { patients }\end{array}$ & $\begin{array}{l}\text { Calcitriol; } \\
\text { paricalcitol }\end{array}$ & $\begin{array}{l}\text { No } \\
\text { treatment }\end{array}$ & $\begin{array}{l}\text { 1.9-3.3 } \\
\text { ug/week; } \\
11.2-15.9 \\
\text { ug/week }\end{array}$ & $\begin{array}{l}\text { Prospective } \\
\text { cohort } \\
\text { multicenter } \\
\text { study }\end{array}$ & $\begin{array}{l}\text { Time-dependent } \\
\text { Cox model }\end{array}$ & 18 & no & 5 \\
\hline Dierkes et al. & 2011 & Germany & 650 & NA & NA & $\begin{array}{l}\text { Calcitrol; } \\
\text { cholecalciferol }\end{array}$ & $\begin{array}{l}\text { No } \\
\text { treatment }\end{array}$ & NA & $\begin{array}{l}\text { Prospective } \\
\text { cohort } \\
\text { multicenter } \\
\text { study }\end{array}$ & NA & 24 & NA & NA \\
\hline Ogawa et al. & 2012 & Japan & 190 & 2005 to 2010 & $\begin{array}{l}\text { Prevalent } \\
\text { HD patients }\end{array}$ & Alfacalcidol & $\begin{array}{l}\text { No } \\
\text { treatment }\end{array}$ & $\begin{array}{l}\text { 3.4-7.0 } \\
\text { ug/week }\end{array}$ & $\begin{array}{l}\text { Prospective } \\
\text { cohort single } \\
\text { center study }\end{array}$ & $\begin{array}{l}\text { Baseline Cox } \\
\text { model }\end{array}$ & 60 & no & 9 \\
\hline
\end{tabular}


(HR) was plotted against its standard error. The Begg rank correlation test was used to examine asymmetry of the funnel plot [22]. The Egger weighted linear regression test was used to examine the association between mean effect estimate and its variance [23]. If an asymmetric funnel plot was found, a contour-enhanced funnel plot was used to further explore the source of bias [24]. $\mathrm{P}<0.05$ was considered statistically significant. All tests were 2 -sided. All analyses were conducted using STATA version 12.0 (StataCorp, College Station, Texas).

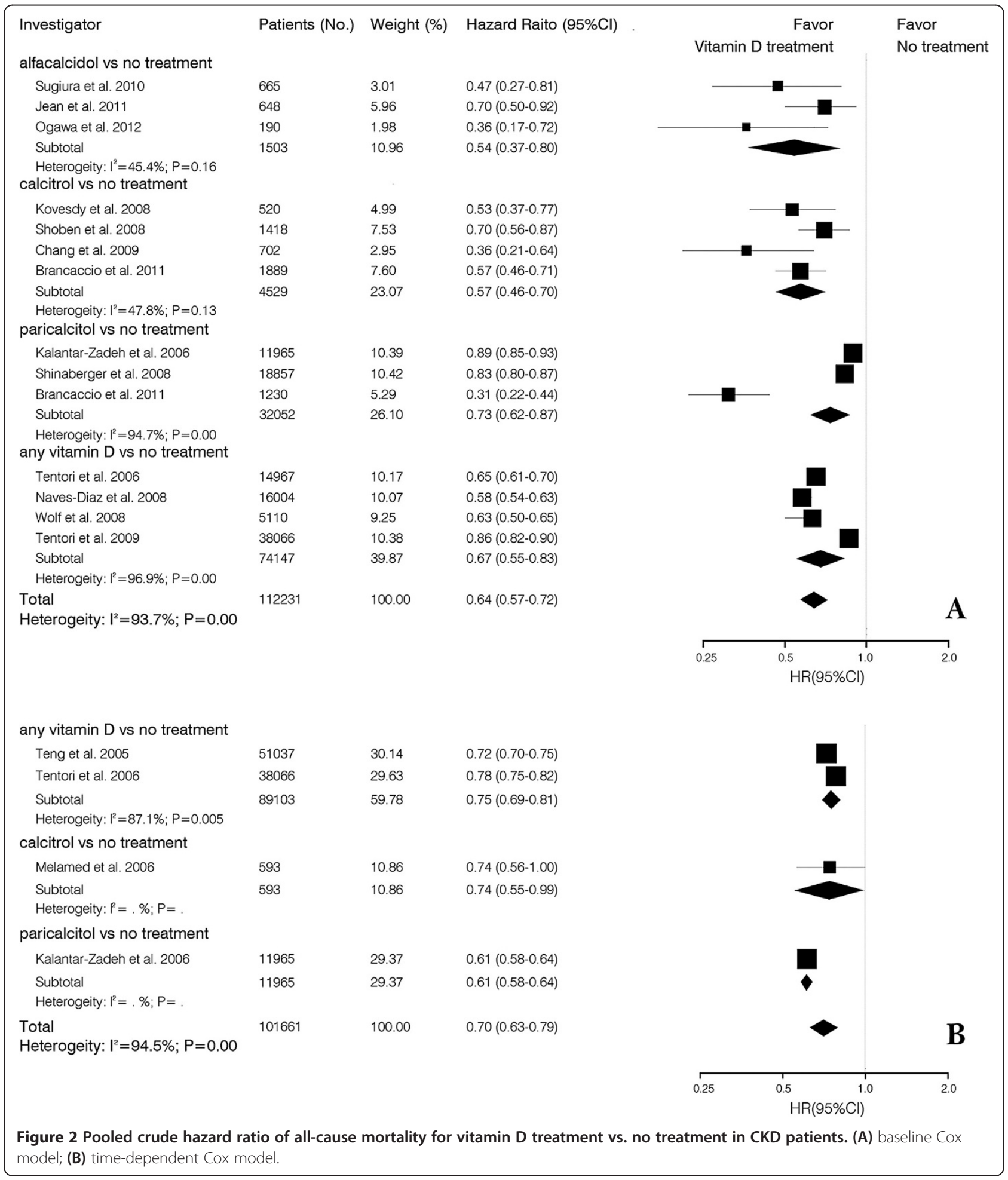




\section{Results}

Literature search

Our initial literature search yielded 2483 citations. 2319 articles were excluded. The majority of these citations were excluded at the level of title or abstract review. There were
164 citations which were considered to be potentially eligible. 144 articles were excluded after reviewing the article. Excluded articles included 37 narrative reviews, 31 duplication studies, 23 without vitamin D treatment, 20 without survival outcome, 15 without survival outcome

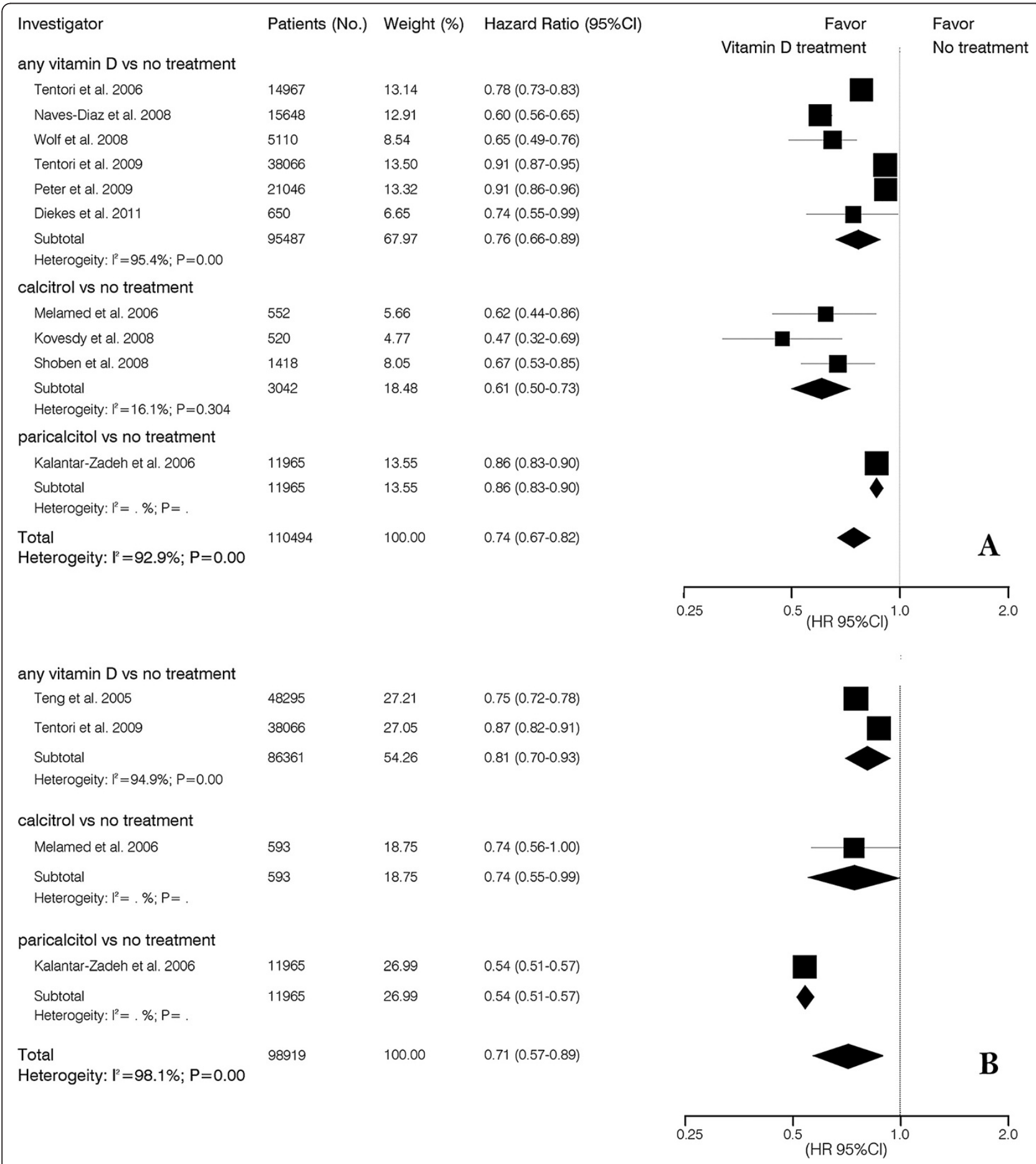

Figure 3 Pooled case mixed adjusted hazard ratio of all-cause mortality for vitamin D treatment vs. no treatment in CKD patients. (A) baseline Cox model; (B) time-dependent Cox model. 
Table 2 Pooled hazard ratio for ESRD on dialysis and CKD not on dialysis

\begin{tabular}{|c|c|c|c|c|}
\hline Patient group & \# patients & Hazard ratio & \# studies & $I^{2}, \%$ \\
\hline \multicolumn{5}{|l|}{ Patients with CKD not on dialysis } \\
\hline Crude all-cause mortality & 2603 & $0.61(0.48-0.77)$ & 3 & 29.2 \\
\hline Adjusted all-cause mortality & 2603 & $0.59(0.35-0.99)$ & 3 & 79 \\
\hline \multicolumn{5}{|l|}{ Patients with ESRD on dialysis } \\
\hline Crude all-cause mortality & 109628 & $0.65(0.58-0.73)$ & 11 & 95 \\
\hline Adjusted all-cause mortality & 66639 & $0.80(0.68-0.94)$ & 6 & 94.4 \\
\hline
\end{tabular}

data, 9 systematic reviews or meta-analyses, 5 author replies, 2 comments, 2 editorials and 1 letter. Twenty studies were considered eligible to be included in the meta-analysis [25-44]. The overall search flow is presented in Figure 1.

\section{Study characteristics}

The characteristics of eligible studies are summarized in Table 1. Of the 20 included observational studies, eleven were prospective cohort studies. [26,28,31,34-36,39,41-44]. The remaining 9 consisted of 6 historical cohort studies $[25,27,29,30,32,33]$ and 3 retrospective cohort studies $[37,38,40]$. Seventeen studies reported ESRD patients on dialysis [25-30,32,33,35-39,41-44] and three reported CKD patients not on dialysis $[31,34,40]$. Five studies compared calcitriol to no active vitamin D treatment $[28,31,34,38,42]$, two studies compared paricalcitol to no active vitamin D treatment $[33,42]$ and four studies compared alfacalcidol to no active vitamin D treatment $[26,40,41,44]$. Nine studies did not report the specific analogues used and compared active vitamin $\mathrm{D}$ compounds with no treatment $[27,29,30,32,35-37,39,43]$. Two studies compared the survival benefits of paricalcitol and calcitriol [25,30]. Several sophisticated statistical models were used in these observational studies. Fifteen studies used a fixed covariate baseline Cox model [25,26,30-35,37-41,43,44], two studies used a time-dependent Cox model $[27,42]$, and three studies used both Cox models $[28,29,36]$. Only 4 studies were confirmed by intention to treat (ITT) analysis [27,30,32,34].

\section{Vitamin D and all-cause mortality}

14 studies examined the association between active vitamin $\mathrm{D}$ treatment and crude all-cause mortality. Patients that received alfacalcidol had a 46\% (HR, 0.54; 95\% CI, 0.37-0.80) lower overall mortality risk compared to untreated patients. Calcitriol, paricalcitol and not otherwise specified active vitamin D treated patients had a 43\% (HR, 0.57; 95\% CI, $0.46-0.70), 27 \%$ (HR, 0.73; 95\% CI, 0.62-0.87) and 36\% (HR, 0.64; 95\% CI, 0.57-0.72) lower overall mortality risk. Similar results were observed with the crude timedependent Cox model. All-cause mortality risk with calcitriol, paricalcitol and not otherwise specified active vitamin D was 26\% (HR, 0.74; 95\% CI, 0.55-0.99), 39\% (HR, 0.61; 95\% CI, 0.58-0.64) and 30\% (HR, 70; 95\% CI, 0.63-0.79) lower, respectively, than that found patients without active vitamin D treatment (Figure 2).

Ten studies reported vitamin D intake and risk for all-cause mortality using an adjusted case mixed baseline model. The risk of all-cause mortality was reduced 39\% (HR, 0.61; 95\% CI, 0.50-0.73) with calcitriol and 14\% (HR, 0.86; 95\% CI, 0.83-0.90) with paricalcitol. Using the adjusted case mixed time-dependent Cox model, patients who received active vitamin $\mathrm{D}$ treatment had a survival benefit (HR, 0.71; 95\% CI, 0.57-0.89) (Figure 3).

Table 3 Pooled hazard ratio for cardiovascular mortality in patients receiving vitamin D or no treatment

\begin{tabular}{|c|c|c|c|c|}
\hline Patient groups & \# patients & Hazard ratio & \# studies & $12, \%$ \\
\hline \multicolumn{5}{|c|}{ Crude cardiovascular mortality using baseline Cox model } \\
\hline Alfacalcitol vs no treatment & 432 & $0.37(0.25-0.55)$ & 2 & 0 \\
\hline Calcitrol vs no treatment & 1889 & $0.57(0.46-0.71)$ & 1 & NA \\
\hline Paricalcitol vs no treatment & 1230 & $0.31(0.22-0.44)$ & 1 & NA \\
\hline Overall & 3551 & $0.41(0.28-0.59)$ & 4 & 69.9 \\
\hline \multicolumn{5}{|c|}{ Adjusted cardiovascular mortality using baseline Cox model } \\
\hline Any vitamin $D$ vs no treatment & 466 & $0.59(0.19-1.82)$ & 2 & 68.6 \\
\hline Alfacalcitol vs no treatment & 665 & $0.45(0.14-1.47)$ & 1 & NA \\
\hline Calcitrol vs no treatment & 1889 & $0.63(0.50-0.79)$ & 1 & NA \\
\hline Paricalcitol vs no treatment & 1230 & $0.43(0.29-0.63)$ & 1 & NA \\
\hline Overall & 4250 & $0.59(0.41-0.86)$ & 5 & 83.6 \\
\hline
\end{tabular}


Table 4 Comparison of all-cause mortality with paricaltitol and calcitrol

\begin{tabular}{lllll}
\hline Patient group & \# patients & Hazard ratio & \# studies & I2, \% \\
\hline Crude baseline Cox model & 75130 & $0.80(0.75-0.86)$ & 2 & 0 \\
Adjusted baseline Cox case mixed model & 16008 & $0.89(0.79-1.00)$ & 3 & 62.9 \\
Adjusted baseline Cox case mixed and MICS model & 14384 & $0.95(0.91-0.99)$ & 2 & 0 \\
\hline
\end{tabular}

We pooled data for ESRD on dialysis patients and CKD not on dialysis patients. Three studies evaluated patients with CKD that were not on dialysis. The survival advantage was similar in both the crude model (HR, 0.61; 95\% CI, 0.43-0.77) and the adjusted model (HR, 0.59; 95\% CI, 0.35-0.99). Patients with ESRD on dialysis had less survival benefit in the adjusted model (HR, 0.80; 95\% CI, 0.63-0.94) than in the crude model (HR, 0.65; 95\% CI, 0.58-0.73) (Table 2).

\section{Vitamin D and cardiovascular mortality}

Four studies reported the HR between active vitamin D treatment and cardiovascular mortality using a crude Cox model and five using an adjusted baseline Cox model. A significant survival advantage was found in patients receiving active vitamin $\mathrm{D}$ using an unadjusted analysis (HR, 0.41; 95\% CI, 0.28-0.59) and an adjusted analysis (HR, 0.59; 95\% CI, 0.41-0.86). Similar results were found with calcitriol and paricalcitol. The adjusted baseline Cox model analysis found the reduction of cardiovascular mortality with calcitriol and paricalcitol to be $37 \%$ (HR, 0.63; 95\% CI, 0.50-0.79) and 57\% (HR, 0.43 ; $95 \%$ CI, 0.29-0.63), respectively. There was no survival difference associated with alfacalcidol treatment (HR, 0.45; 95\% CI, 0.14-1.47) (Table 3).

\section{Calcitriol vs paricalcitol and all-cause mortality}

Three studies reported hazard ratios that compared calcitriol and paricalcitol treatment. The crude baseline Cox model found a survival advantage with paricalcitol treatment (HR, 0.80; 95\% CI, 0.75-0.86). In contrast, the adjusted baseline Cox case mixed and malnutrition-inflammation-cachexia syndrome (MICS) model demonstrated a survival advantage with calcitriol treatment (HR, 0.95; 95\% CI, 0.91-0.99) in (Table 4).

\section{Vitamin D dosage and all-cause mortality}

Three studies reported the relationship between active vitamin $\mathrm{D}$ dose and all-cause mortality. Calcitriol treatment was associated with a dose dependent decrease in all-cause mortality. There was no survival advantage when calcitriol dose exceeded 7 ug per week. A dose dependent response was not found with paricalcitol (Table 5).

\section{Assessment bias and meta-regression analysis}

A publication bias was identified using an Egger regression asymmetry test $(\beta=-3.81, P=0.01)$ and a funnel plot (Figure 4). A contour-enhanced funnel plot was used to explore the source of the bias. The contour-enhanced funnel plot demonstrated that the majority of studies

Table 5 Vitamin D dosage and all-cause mortality risk

\begin{tabular}{|c|c|c|c|c|c|c|}
\hline Investigator & \# patients & $\begin{array}{l}\text { Follow up } \\
\text { (months) }\end{array}$ & $\begin{array}{l}\text { Dosage } \\
\text { (ug/week) }\end{array}$ & $\begin{array}{l}\text { Mean dosage } \\
\text { (ug/week) }\end{array}$ & Hazard ratio & $95 \% \mathrm{Cl}$ \\
\hline \multicolumn{7}{|l|}{ Calcitrol } \\
\hline Naves-Diaz et al. & 1304 & 54 & $<1.75$ & 1.05 & 0.46 & $0.37-0.53$ \\
\hline Naves-Diaz et al. & 1053 & 54 & $1.75-3.5$ & 2.38 & 0.58 & $0.49-0.70$ \\
\hline Naves-Diaz et al. & 432 & 54 & $3.5-7.0$ & 4.69 & 0.64 & $0.50-0.83$ \\
\hline Naves-Diaz et al. & 184 & 54 & $>7.0$ & 11.83 & 0.83 & $0.58-1.19$ \\
\hline \multicolumn{7}{|l|}{ Paricalcitol } \\
\hline Kalantar-Zadeh et al. & 5288 & 24 & $1.0-5.0$ & NA & 0.53 & $0.50-0.57$ \\
\hline Kalantar-Zadeh et al. & 11965 & 24 & $5.0-10.0$ & NA & 0.54 & $0.51-0.57$ \\
\hline Kalantar-Zadeh et al. & 8326 & 24 & $10.0-15.0$ & NA & 0.54 & $0.51-0.57$ \\
\hline Kalantar-Zadeh et al. & 11816 & 24 & $>15.0$ & NA & 0.73 & $0.69-0.77$ \\
\hline Shinaberger et al. & 9575 & 30 & $1.7-20.1$ & 10.9 & 0.93 & $0.89-0.97$ \\
\hline Shinaberger et al. & 8277 & 30 & $4.6-25.8$ & 15.2 & 0.88 & $0.84-0.94$ \\
\hline Shinaberger et al. & 5875 & 30 & $6.4-30.8$ & 18.6 & 0.88 & $0.84-0.93$ \\
\hline
\end{tabular}



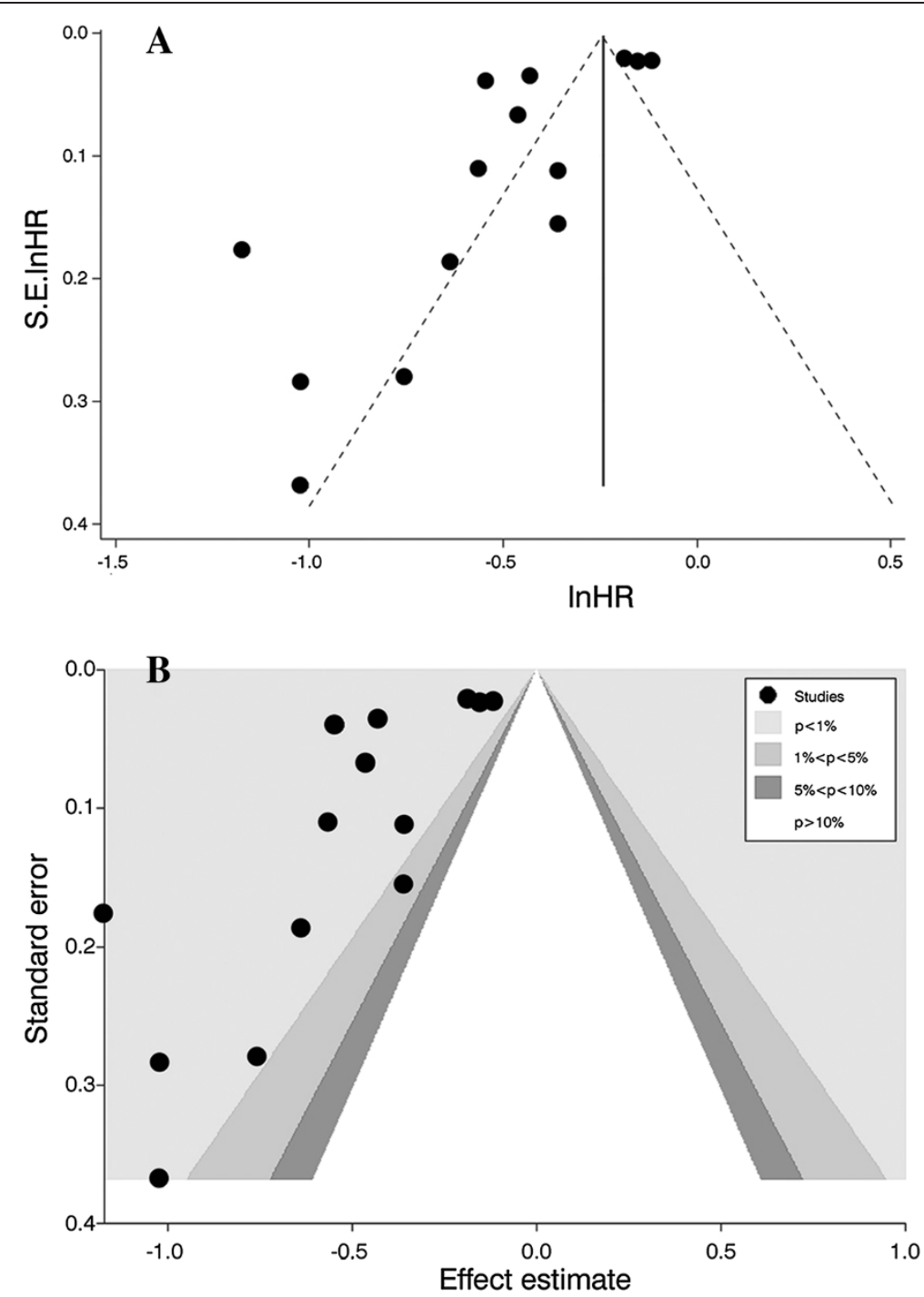

Figure 4 Funnel plot and contour-enhanced funnel plot used to explore the source of publication bias. (A) funnel plot;

(B) contour-enhanced funnel plot.

had a high statistical significance. Therefore, publication bias was a less likely cause of the funnel plot asymmetry (Figure 4).

Within study heterogeneity was evaluated using covariate meta regression analysis. Of the seven covariates, publication year $(\mathrm{t}=-2.19, \mathrm{P}=0.049)$ and study participants $(t=2.52, P=0.027)$ had the greatest between study variance. The proportion of within-study variance explained by publication year and study participants was $24.14 \%$ and $36.20 \%$, respectively (Figure 5 ).

\section{Discussion}

Active vitamin D compounds were associated with a reduced risk of mortality in patients with ESRD on dialysis and patients with CKD not requiring dialysis. Several mechanisms may explain how vitamin D can modify risk for mortality. Vitamin D down regulates the reninangiotensin system [45], improves insulin secretion and sensitivity [46], inhibits vascular smooth-muscle cell proliferation [47], protects normal endothelial cell function [48], modulates inflammatory processes [49], inhibits anticoagulant activity [50], and inhibits myocardial cell hypertrophy and proliferation [51]. These findings suggest that vitamin $\mathrm{D}$ may decrease mortality through multiple pathways. Although the actual mechanism of mortality is unclear, patient death has been associated with vascular calcifications, left-ventricular hypertrophy and leftventricular dysfunction. The multi-organ protective effects of vitamin D may explain the lower mortality rate found in these patients.

A fixed covariate baseline Cox model was used in the majority of included studies. Only 5 studies used a time- 

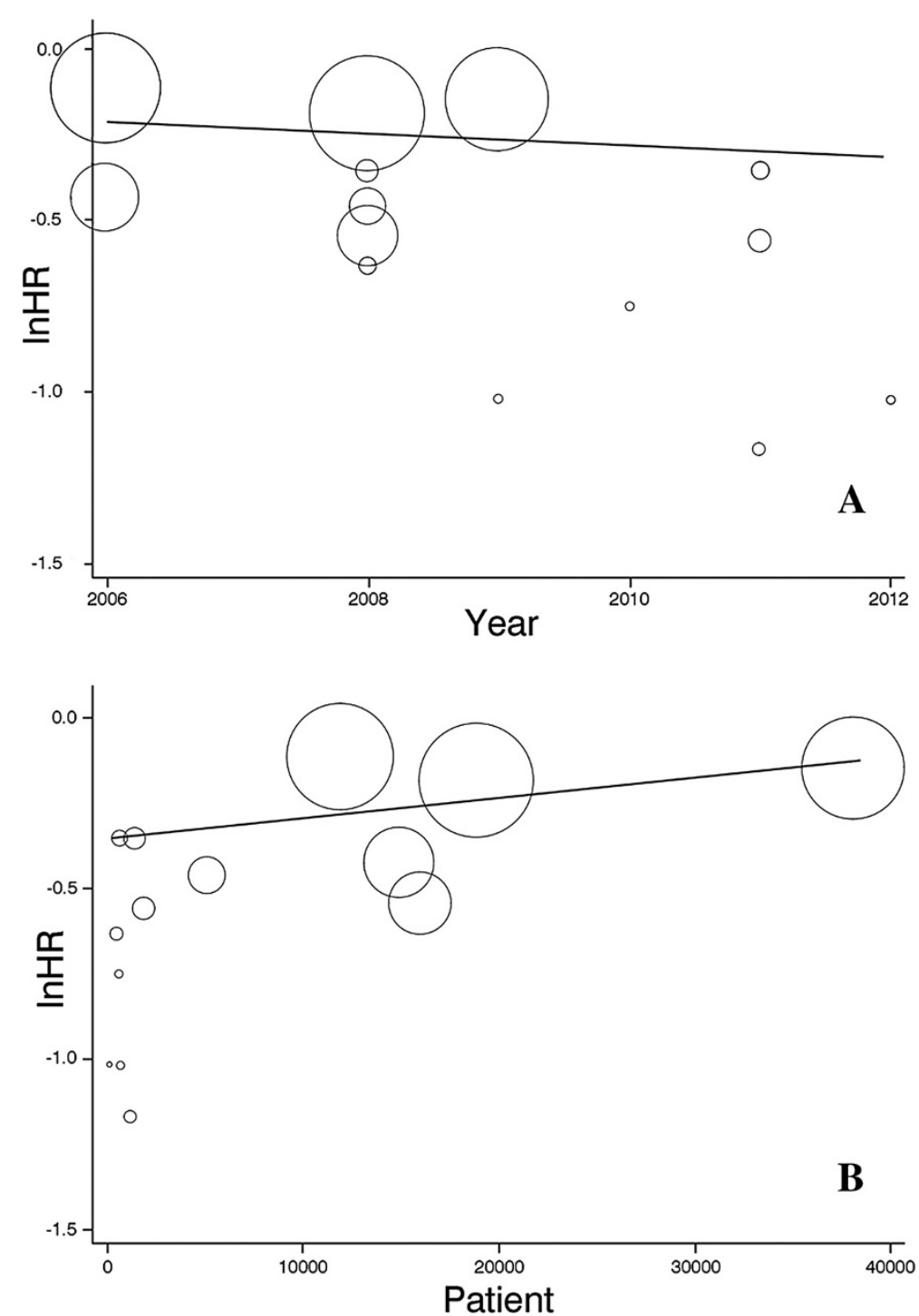

Figure 5 Meta-regression graph of hazard ratio for all-cause mortality in vitamin $D$ treated vs. no treatment patients.

(A) meta-regression by publication year; (B) meta-regression by number of study patients.

dependent Cox model to analyze the relationship between active vitamin D use and survival. Although a standard baseline Cox proportional regression model is usually used to analyze cohort studies, it may be inadequate to evaluate active vitamin $\mathrm{D}$ treatments due to the presence of time-dependent variation in outcome. Higher serum calcium and phosphorus levels were consistently associated with increased risk of death [4,52]. Elevated serum PTH levels have also been associated with increased mortality $[4,33]$. The serum levels of calcium, phosphorus and PTH are affected by vitamin D therapy. Serum levels of calcium, phosphorus and PTH vary during the course of vitamin D therapy and affect patient outcome. These mineral metabolism indexes are recognized as time-dependent confounders. Time-dependent confounders cannot be controlled by conventional survival analysis methods [53]. Marginal structural modeling (MSM) can control for time-dependent confounders affected by prior treatment [54]. Under some conditions, the treatment estimate from a MSM can have the same causal interpretation as an estimate from a randomized clinical trial [55]. Only the Tentori et al. study reported detailed data regarding the survival advantage of patients treated with active vitamin D. The unadjusted baseline Cox model and time-varying MSM models demonstrated a $16 \%$ and $22 \%$, respectively, reduction of all-cause mortality associated with active vitamin D treatment. Most studies included in this meta-analysis had some selection bias. For example, the study of Teng et al. [27] had statistical differences in the baseline characteristics of patient age, primary cause of 
renal failure, body mass index, blood pressure, and intact parathyroid hormone and hemoglobin levels. Several studies included in the meta-analysis used sophisticated statistical techniques, such as adjustment for time-dependent confounders, propensity score-matching or marginal structural models, to mimic the design of randomized controlled trials. Only the characteristics of patients that were treated with vitamin $\mathrm{D}$ analogues were known to the researchers. Any confounding factors would be controlled by these statistical methods and the results would be comparable to randomized controlled trials. The problem with the observational studies was that such knowledge was not available. The potential presence of unmeasured confounders prevented any conclusions of causation, even when sophisticated statistical methods were used. The survival advantage associated with active vitamin D treatment occurred in a dose-dependent manner. This phenomenon has been supported by two studies [29,32]. There has been no welldesigned dose gradient study to test this hypothesis. Although we do not have higher quality evidence to prove this association, we believe that vitamin D will improve survival.

The meta-analysis detected slight differences in survival associated with different analogues of active vitamin D. The baseline case mixed and MICS Cox models detected a $5 \%$ lower mortality with paricalcitol treatment than with calcitriol treatment. This slight survival difference may be explained by differential effects of calcitriol and its analogue, paricalcitol on vascular calcification. In vitro studies have demonstrated that calcitriol is a growth factor for vascular smooth muscle cells, while the analogue, paricalcitol, is not [56]. In vivo studies have shown that vitamin $\mathrm{D}$ sterols have a differential effect on vascular calcification. 1- $\alpha$-hydroxy vitamin D (calcitriol) was associated with greater vascular calcification than paricalcitol, even though there was equivalent suppression of PTH in these animal models [57]. Only two well-designed cohort studies or randomized controlled trials, Teng et al. [25] and Tentori et al. [30], have evaluated the mortality risk associated with different active vitamin D analogues. Further studies are needed to clarify the survival difference before one vitamin $\mathrm{D}$ analogue is recommended over another in clinical practice.

Three studies included in the meta-analysis reported mortality risk associated with different mean daily or weekly doses of vitamin D. In the Naves-Diaz et al. study, the maximum reduction of mortality occurred when the mean daily dose of oral calcitriol was less than $0.25 \mathrm{ug}$. This survival benefit was lost as the mean daily calcitriol dose was increased to more than 1.0 ug. This dosedependent benefit effect was also reported with paricalcitol. Kalantar-Zadeh et al. reported patients treated with mean weekly doses of $1.0 \mathrm{ug}$ to $5.0 \mathrm{ug}$ of paricalcitol. Mean weekly doses of paricalcitol above 15.0 ug were associated with an $18 \%$ reduction of mortality risk. A possible explanation is that low-dose vitamin D exerts weaker anti-vascular calcification effects than higher doses in CKD patients. High doses of vitamin D could be associated with adverse effects, such as hypercalcemia, that would overwhelm its protective effects.

There were several limitations in our meta-analysis. First, only a few of the included studies used a time-dependent or marginal structural model to analyze the follow-up data. The majority of studies had limited power to draw a definitive conclusion on the effects of vitamin D supplements on all-cause or cardiovascular mortality. Second, there was high heterogeneity in the meta-analysis. Sample size and publication year were the sources of heterogeneity. Third, the possible sources of heterogeneity could not be carefully examined. This included observational studies of the use of recombinant erythropoietin to correct anemia and studies of phosphorus binders to ameliorate hyperphosphatemia in patients with CKD that showed beneficial effects on mortality, CVD outcome, and progression of renal disease. Fourth, we did not seek to identify unpublished studies and several studies were excluded because the published data were not suitable for meta-analysis.

\section{Conclusions}

Active vitamin D compounds used to treat abnormal calcium, phosphorus and PTH levels in patients with either ESRD on dialysis or CKD not requiring dialysis. Active vitamin D compound treatment was associated with decreased all cause and cardiovascular mortality. Low dose active vitamin D compounds were associated with improved survival. Large, well designed randomized trials of active vitamin D supplements with different doses are needed to elucidate the role of vitamin D supplementation in reducing mortality.

\section{Competing interests}

The authors declare that they have no competing interests.

\section{Authors' contributions}

$\mathrm{ZZ}$ and HS are responsible for the literature assessment and data analysis. J J and DL contributed to the literature search and manuscript drafts. SL was a designer of entire study. All authors read and approved the final manuscript.

\section{Acknowledgements}

We thank doctor Yang Xue for manuscript and figure editing.

\section{Author details}

${ }^{1}$ Nephrology Department, General Hospital of Tianjin Medical University, No. 154 Anshan Road, Heping District, Tianjin 300052, China. ${ }^{2}$ Radiology Department, General Hospital of Tianjin Medical University, No.154 Anshan Road, Heping District, Tianjin 300052, China.

Received: 12 May 2013 Accepted: 4 September 2013 Published: 25 September 2013

\section{References}

1. Moe S, Drueke T, Cunningham J, Goodman W, Martin K, Olgaard K, Ott S, Sprague S, Lameire N, Eknoyan G, et al: Definition, evaluation, and classification of renal osteodystrophy: a position statement from Kidney Disease: Improving Global Outcomes (KDIGO). Kidney Int 2006, 69:1945-1953. 
2. Goodman WG, Goldin J, Kuizon BD, Yoon C, Gales B, Sider D, Wang Y, Chung J, Emerick A, Greaser L, et al: Coronary-artery calcification in young adults with end-stage renal disease who are undergoing dialysis. N Engl J Med 2000, 342:1478-1483.

3. Qunibi WY, Nolan CA, Ayus JC: Cardiovascular calcification in patients with end-stage renal disease: a century-old phenomenon. Kidney Int 2002, 62(Suppl):73-80.

4. Block GA, Klassen PS, Lazarus JM, Ofsthun N, Lowrie EG, Chertow GM: Mineral metabolism, mortality, and morbidity in maintenance hemodialysis. J Am Soc Nephrol 2004, 15:2208-2218.

5. De Boer I, loannou GN, Kestenbaum B, Brunzell JD, Weiss NS 25(OH)D levels and albuminuria in the Third National Health and Nutrition Examination Survey (NHANES III). Am J Kidney Dis 2007, 50:69-77

6. Forman JP, Giovannucci E, Holmes MD, Bischoff-Ferrari HA, Tworoger SS, Willett WC, Curhan GC: Plasma 25(OH)D levels and risk of incident hypertension. Hypertension 2007, 49:1063-1069.

7. Pittas AG, Dawson-Hughes B, Li T, Van Dam RM, Willett WC, Manson JE, Hu FB: Vitamin $D$ and calcium intake in relation to type 2 diabetes in women. Diabetes Care 2006, 29:650-656.

8. Tentori F, Blayney MJ, Albert JM, Gillespie BW, Kerr PG, Bommer J, Young EW, Akizawa T, Akiba T, Pisoni RL, et al: Mortality risk for dialysis patients with different levels of serum calcium, phosphorus, and PTH: The Dialysis Outcomes and Practice Patterns Study (DOPPS). Am J Kidney Dis 2008, 52:519-530.

9. Kovesdy CP, Ahmadzadeh S, Anderson JE, Kalantar-Zadeh K: Secondary hyperparathyroidism is associated with higher mortality in men with moderate to severe chronic kidney disease. Kidney Int 2008, 73:1296-1302.

10. Artaza JN, Mehrotra R, Norris KC: Vitamin D and cardiovascular system. Clin J Am Soc Nephrol 2009, 4:1515-1522.

11. Wang L, Manson JE, Song Y, Sesso HD: Systematic review: Vitamin D and calcium supplementation in prevention of cardiovascular events. Ann Intern Med 2010, 152:315-323.

12. Pittas AG, Chung M, Trikalinos T, Brendel M, Patel K, Lichtenstein AH, Lau J, Balk EM: Systematic review: Vitamin D and cardiometabolic outcomes. Ann Intern Med 2010, 152:307-314.

13. Kandula P, Dobre M, Schold JD, Schreiber MJ, Mehrotra R, Navaneethan SD: Vitamin D supplementaion in chronic kidney disease: A systematic review and meta-analysis of observational studies and randomized controlled trials. Clin J Am Soc Nephrol 2011, 6:50-62.

14. Dickersin K, Scherer R, Lefebvre C: Identifying relevant studies for systematic reviews. BMJ 1994, 309(6964):1286-1291.

15. Lefebvre C, McDonald S: Development of sensitive search strategy for reports of randomized controlled trials in EMBASE. In Fourth International Cochrane Colloquium: Oct 20-24 1996; Adelaide. Adelaide (Australia): The Australian Cochrane Centre; 1996.

16. Stroup DF, Berlin JA, Morton SC, Olkin I, Williamson GD, Rennie D, Moher D Becker BJ, Sipe TA, Thacker SB: Meta-analysis Of Observational Studies in Epidemiology (MOOSE) Group. Meta-analysis of observational studies in epidemiology. JAMA 2000, 283(15):2008-2012.

17. Wells GA, Shea B, O'Connell D, Peterson J, Welch V, Losos M, Tugwell P: The Newcastle-Ottawa Scale (NOS) for assessing the quality of nonrandomized studies in meta-analysis. Ottawa, Canada: Department of Epidemiology and Community Medicine, University of Ottawa; 2010. http://www.ohri.ca/ programs/clinical_epidemiology/oxford.asp.

18. von Elm E, Altman DG, Egger M, Pocock SJ, Gøtzsche PC, Vandenbroucke JP: STROBE Initiative: Strengthening the Reporting of Observational Studies in Epidemiology (STROBE) statement: guidelines for reporting observational studies. BMJ 2007, 335(7624):806-808.

19. Higgins JPT, Thompson SG: Quantifying heterogeneity in a meta-analysis. Stat Med 2002, 21(11):1539-1558.

20. Huedo-Medina TB, Sánchez-Meca J, Marín-Martínez F, Botella J: Assessing heterogeneity in meta-analysis: Q statistic or $\mathrm{I}^{2}$ index? Psychol Methods 2006, 11(2):193-206

21. Higgins JP, Thompson SG, Deeks JJ, Altman DG: Measuring inconsistency in meta-analysis. BMJ 2003, 327(7417):557-560.

22. Begg CB, Mazumdar M: Operating characteristics of a rank correlation test for publiction bias. Biometrics 1994, 50(4):1088-1101.

23. Egger M, Davey SG, Schneider M, Minder C: Bias in meta-analysis detected by a simple graphical test. BMJ 1997, 315(7109):629-634
24. Peters JL, Sutton AJ, Jones DR, Abrams KR, Rushton L: Contour-enhanced meta-analysis funnel plots help distinguish publication bias from other causes of asymmetry. J Clin Epidemiol 2008, 61(10):991-996.

25. Teng $M$, Wolf $M$, Lowrie $E$, Ofsthun $N$, Lazarus JM, Thadhani R: Survival of patients undergoing hemodialysis with paricalcitol or calcitriol therapy. N Engl J Med 2003, 349(5):446-456.

26. Shoji T, Shinohara K, Kimoto E, Emoto M, Tahara H, Koyama H, Inaba M, Fukumoto S, Ishimura E, Miki T, et al: Lower risk for cardiovascular mortality in oral 1alpha-hydroxy vitamin D3 users in a haemodialysis population. Nephrol Dial Transplant 2004, 19(1):179-184.

27. Teng M, Wolf M, Ofsthun MN, Lazarus JM, Hernán MA, Camargo CA Jr, Thadhani R: Activated injectable vitamin D and hemodialysis survival: a historical cohort study. J Am Soc Nephrol 2005, 16(4):1115-1125.

28. Melamed ML, Eustace JA, Plantinga L, Jaar BG, Fink NE, Coresh J, Klag MJ, Powe NR: Changes in serum calcium, phosphate, and PTH and the risk of death in incident dialysis patients: a longitudinal study. Kidney Int 2006, 70(2):351-357

29. Kalantar-Zadeh K, Kuwae N, Regidor DL, Kovesdy CP, Kilpatrick RD, Shinaberger CS, McAllister CJ, Budoff MJ, Salusky IB, Kopple JD: Survival predictability of time-varying indicators of bone disease in maintenance hemodialysis patients. Kidney Int 2006, 70(4):771-780.

30. Tentori F, Hunt WC, Stidley CA, Rohrscheib MR, Bedrick EJ, Meyer KB, Johnson HK, Zager PG, Medical Directors of Dialysis Clinic Inc: Mortality risk among hemodialysis patients receiving different vitamin $D$ analogs. Kidney Int 2006, 70(10):1858-1865.

31. Kovesdy CP, Ahmadzadeh S, Anderson JE, Kalantar-Zadeh K: Association of activated vitamin $\mathrm{D}$ treatment and mortality in chronic kidney disease. Arch Intern Med 2008, 168(4):397-403.

32. Naves-Díaz M, Alvarez-Hernández D, Passlick-Deetjen J, Guinsburg A, Marelli C, Rodriguez-Puyol D, Cannata-Andía JB: Oral active vitamin D is associated with improved survival in hemodialysis patients. Kidney Int 2008, 74(8):1070-1078.

33. Shinaberger CS, Kopple JD, Kovesdy CP, McAllister CJ, van Wyck D, Greenland S, Kalantar-Zadeh K: Ratio of paricalcitol dosage to serum parathyroid hormone level and survival in maintenance hemodialysis patients. Clin J Am Soc Nephrol 2008, 3(6):1769-1776.

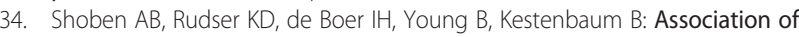
oral calcitriol with improved survival in nondialyzed CKD. J Am SoC Nephrol 2008, 19(8):1613-1619.

35. Wolf M, Betancourt J, Chang Y, Shah A, Teng M, Tamez H, Gutierrez O, Camargo CA Jr, Melamed M, Norris K, et al: Impact of activated vitamin D and race on survival among hemodialysis patients. J Am Soc Nephrol 2008, 19(7):1379-1388.

36. Tentori F, Albert JM, Young EW, Blayney MJ, Robinson BM, Pisoni RL, Akiba T, Greenwood RN, Kimata N, Levin NW, et al: The survival advantage for haemodialysis patients taking vitamin $D$ is questioned: findings from the Dialysis Outcomes and Practice Patterns Study. Nephrol Dial Transplant 2009, 24(3):963-972.

37. St Peter WL, Li S, Liu J, Gilbertson DT, Arneson TJ, Collins AJ: Effects of monthly dose and regular dosing of intravenous active vitamin $D$ use on mortality among patients undergoing hemodialysis. Pharmacotherapy 2009, 29(2):154-164

38. Chang $\mathrm{HC}$, Chen $\mathrm{CL}$, Chiu TL, Chen SI, Yen AM, Chen TH: Effectiveness of recombinant human erythropoietin, vitamin D3 and iron therapy on long-term survival of patients with end-stage renal disease receiving haemodialysis: analysis of 702 patients after 10-year follow-up. Public Health Nutr 2009, 12(12):2410-2415.

39. Konta T, Hoshikawa M, Takasaki S, Ichikawa K, Mashima Y, Takahashi T, Abiko $H$, Masakane I, Matsunaga T, Suzuki M, et al: Patient survival and the combination of vitamin D and phosphorus binder in dialysis. Dialysis and Transplantation 2010, 39(10):427-431.

40. Sugiura S, Inaguma D, Kitagawa A, Murata M, Kamimura Y, Sendo S, Hamaguchi K, Nagaya H, Tatematsu M, Kurata K, et al: Administration of alfacalcidol for patients with predialysis chronic kidney disease may reduce cardiovascular disease events. Clin Exp Nephrol 2010, 14(1):43-50.

41. Jean G, Lataillade D, Genet L, Legrand E, Kuentz F, Moreau-Gaudry X, Fouque D: ARNOS Study Investigators: Impact of hypovitaminosis D and alfacalcidol therapy on survival of hemodialysis patients: results from the French ARNOS study. Nephron Clin Pract 2011,

118(2):c204-c210 
42. Brancaccio D, Cozzolino M, Cannella G, Messa P, Bonomini M, Cancarini G, Caruso MR, Cascone C, Costanzo AM, di Luzio PU, et al: Secondary hyperparathyroidism in chronic dialysis patients: results of the Italian FARO survey on treatment and mortality. Blood Purif 2011, 32(2):124-132.

43. Dierkes J, Kropf S, Machlitt P, Domroese U, Neumann K, Westphal S, Luley C, Heinz J: Vitamin D supplementation and mortality in patients with endstage renal disease. Ann Nutr Metab 2011, 58(Suppl 3):326.

44. Ogawa M, Ogawa T, Inoue T, Otsuka K, Nitta K: Effect of alfacalcidol therapy on the survival of chronic hemodialysis patients. Ther Apher Dial 2012, 16(3):248-253.

45. Rammos $G$, Tseke P, Ziakka S: Vitamin D, the renin-angiotensin system, and insulin resistance. Int Urol Nephrol 2008, 40:419-426.

46. Chiu KC, Chu A, Go VL, Saad MF: Hypovitaminosis D is associated with insulin resistance and beta cell dysfunction. Am J Clin Nutr 2004, 79:820-825.

47. Carthy EP, Yamashita W, Hsu A, Ooi BS: 1,25-Dihydroxyvitamin D3 and rat vascular smooth muscle cell growth. Hypertension 1989, 13:954-959.

48. Levin A, Li YC: Vitamin D and its analogues: do they protect against cardiovascular disease in patients with kidney disease? Kidney Int 2005, 68:1973-1981

49. Gysemans CA, Cardozo AK, Callewaert H, Giulietti A, Hulshagen L, Bouillon R, Eizirik DL, Mathieu C: 1,25-Dihydroxyvitamin D3 modulates expression of chemokines and cytokines in pancreatic islets: implications for prevention of diabetes in nonobese diabetic mice. Endocrinology 2005, 146:1956-1964.

50. Ohsawa M, Koyama T, Yamamoto K, Hirosawa S, Kamei S, Kamiyama R: 1,25-dihydroxyvitamin $\mathrm{D}(3)$ and its potent synthetic analogs downregulate tissue factor and upregulate thrombomodulin expression in monocytic cells, counteracting the effects of tumor necrosis factor and oxidized LDL. Circulation 2000, 102:2867-2872.

51. Simpson RU, Hershey SH, Nibbelink KA: Characterization of heart size and blood pressure in the vitamin D receptor knockout mouse. J Steroid Biochem Mol Biol 2007, 103:521-524.

52. Young EW, Albert JM, Satayathum S, Goodkin DA, Pisoni RL, Akiba T, Akizawa T, Kurokawa K, Bommer J, Piera L, et al: Predictors and consequences of altered mineral metabolism: the Dialysis Outcomes and Practice Patterns Study. Kidney Int 2005, 67:1179-1187.

53. Robins JM, Hernan MA, Brumback B: Marginal structural models and causal inference in epidemiology. Epidemiology 2000, 11:550-560

54. Robins JM: Correction for non-compliance in equivalence trials. Stat Med 1998, 17:269-302.

55. Cole SR, Hernan MA, Robins JM, Anastos K, Chmiel J, Detels R, Ervin C, Feldman J, Greenblatt R, Kingsley L, et al: Effect of highly active antiretroviral therapy on time to acquired immunodeficiency syndrome or death using marginal structural models. Am J Epidemiol 2003, 158:687-694.

56. Cardus A, Panizo S, Parisi E, Fernandez E, Valdivielso JM: Differential effects of vitamin D analogs on vascular calcifiction. J Bone Miner Res 2007, 22:860-866.

57. Mizobuchi M, Finch JL, Martin DR, Slatopolsky E: Differential effects if vitamin $\mathrm{D}$ receptor activators on vascular calcification in uremic rats. Kidney Int 2007, 72:709-715.

\section{doi:10.1186/1471-2369-14-199}

Cite this article as: Zheng et al:: Vitamin D supplementation and mortality risk in chronic kidney disease: a meta-analysis of 20 observational studies. BMC Nephrology 2013 14:199.

\section{Submit your next manuscript to BioMed Central and take full advantage of:}

- Convenient online submission

- Thorough peer review

- No space constraints or color figure charges

- Immediate publication on acceptance

- Inclusion in PubMed, CAS, Scopus and Google Scholar

- Research which is freely available for redistribution 